\title{
Simulation of the breakup channel in three-particle collisions with pseudoreaction channels
}

\author{
Zeki C. Kuruoğlu \\ Chemistry Department, Bilkent University, Ankara,Turkey
}

(Received 20 March 1991)

\begin{abstract}
A pseudochannel extension of the coupled-reaction-channel (CRC) method is investigated formally and numerically as a means of simulating the effect of the breakup channel on the rearrangement amplitudes. The equations of the pseudostate-augmented CRC method are derived within a twoHilbert-space approach. The connection of the effective CRC transition operators with the postform transition operators of the formal scattering theory is established. Alternative formulations of the method corresponding to different ways of treating the nonorthogonality interaction are given. Convergence with respect to the number and nature of the pseudostates is investigated numerically on a solvable three-particle model. It is observed that the results obtained with six distinct sets of pseudochannels that have considerably different threshold energies converge toward the exact ones.
\end{abstract}

PACS number(s): 34.10.+x 03.65.Nk 11.80.Fv 11.80.Jy

\section{INTRODUCTION}

At present, the only foolproof method for treating the three-particle scattering problem above the breakup threshold is the momentum-space integralequation method based on the Faddeev formalism [1, 2]. The moving logarithmic singularities in the kernel of these integral equations are difficult, although not impossible, to handle numerically $[2,3]$. On the other hand, numerical solution of the Schrödinger equation (or the Faddeev equation) in coordinate space with multidimensional methods like finite differences or finite elements are beset by problems involved in the formulation of computationally tractable boundary conditions for the breakup channel $[4,5]$. The standard form of the breakup boundary conditions is obtained by a stationary-phase approximation, and is in the form of outgoing waves in hyperspherical radius. However, recent investigations show that for the stationary-phase approximation to be accurate the hyperradius might have to be excessively large compared to the ranges of the underlying potentials $[5,6]$. The need for a very large hyperradius when the breakup channel is open is also evident in the calculations of Ref. [7] on a collinear model.

In this paper, we consider, in the context of the threeparticle problem, an approximate method in which the breakup channel is simulated by pseudoreaction channels. The pseudochannel concept has been used in the past in various types of collision problems: electron-atom collisions [8], certain models involving a single rearrangement and breakup [9-11], and deuteron-nucleus collisions [12]. The method considered in this article is based on the coupled-reaction-channel (CRC) method [13], which has proven to be very successful in handling the rearrangement dynamics below the breakup threshold [14]. In CRC, the Schrödinger equation is solved on an approximation space, which by construction can accommodate the boundary conditions for the rearrangement channels. For each rearrangement, an approximation subspace is built from direct products of two-particle bound states with a complete set of relative-motion states for the third (spectator) particle. The full approximation space is then the linear span of all rearrangement subspaces. Note that the approximation subspaces for different rearrangements are not orthogonal, but linear independence can usually be ensured, unless there is great disparity between the masses of the three particles involved.

A natural extension of the CRC method would be to augment the standard approximation space with a breakup subspace that can accommodate the breakup boundary conditions. A subspace spanned by direct products of a finite set of functions in hyperangular variables with a complete set of functions in hyperradius (or hypermomentum) would be suitable for this purpose. A time-dependent wave-packet method based on this idea has recently been discussed by the present author [15]. The mathematical foundations of such an approach has been laid out, some time ago, by Chandler and Gibson in their two-Hilbert-space theory [16]. Note that, in this extension of the CRC method, the possibility of linear dependence between the breakup subspace and the rearrangement subspaces can be difficult to avoid. Although rearrangement subspaces are mutually orthogonal, at least, asymptotically, the breakup subspace is not orthogonal to a rearrangement subspace even asymptotically. This difficulty can, in principle, be cured by appropriate pseudoinverse techniques [16-18], but their utility in practice remains to be seen.

In this paper, the usual CRC space is enlarged by augmenting the two-particle bound states with pseudostates, which are square-integrable states embedded in the continua of the two-particle subsystems. The pseudostates in question are obtained by diagonalizing the internal Hamiltonians of the two-particle subsystems in finite subspaces. In the three-particle context, these pseudostates define pseudorearrangement channels and simulate the breakup channel. This approach has the advantage of using only the standard Jacobi variables, and thus reducing the possibility of linear dependence between various subspaces. 
However, the manifold of pseudochannels is not invariant under the the total kinetic-energy operator. Hence, the exact breakup boundary conditions cannot be satisfied $[16,17]$. Thus, the approximate scattering theory developed on the pseudostate-extended CRC space will necessarily involve an approximation of the exact boundary conditions. Energetically accessible pseudoreaction channels are required to satisfy the standard two-fragment, boundary conditions. This approximation defines a consistent and unitary scattering system, but its connection to the original scattering problem has to be established. There are no formal proofs about the convergence of this approximate scattering theory towards the exact one as the number of pseudostates increases. However, numerical studies $[10,19]$ indicate that the approximate theory gives the rearrangement amplitudes accurately, a point further corraborated by the results of calculations presented in the present paper. Note that below the breakup threshold this formal difficulty disappears, for the sole function of the pseudostates in that case is to enlarge the approximation space without any need for approximating the form of the boundary conditions.

Another shortcoming of the pseudochannel idea is that the pseudostates are, to a large extent, arbitrary. By changing the size and the spatial support of the twoparticle $L^{2}$ subspaces that are used to define the pseudostates, the thresholds for pseudochannels as well as the spatial support of the full approximation space can be varied at will. One of the aims of the present paper is to numerically investigate the consequences of the arbitrarines involved in the choice of the pseudostates. In this study, we employ several sets of pseudostates with rather different energy spectra and spatial support, and find that the effect of breakup on reaction amplitudes is indeed predicted correctly, quite independently of the set of pseudostates used.

The pseudochannel extension of the CRC is formulated using the techniques adapted from the two-Hilbert-space approach [16,17,20,21]. The two-Hilbert space approach to scattering theory emphasizes the space of asymptotic states as the main vehicle of specifying a scattering system. In approximate theories, the dynamical content (i.e., channel structure and the corresponding boundary conditions) is fixed through the choice of an asymptotic state space $\mathcal{S}_{\text {as }}$. The full approximation space $\mathcal{S}$ is then the linear span of all states contained in the asymptotic space. Our procedure differs slightly from those of Chandler and Gibson $[16,19]$ in that , instead of $\mathcal{S}_{\text {as }}$, we work directly with an asymptotic spectator space $\mathcal{S}^{\text {as }}$. By setting up a correspondence between $\mathcal{S}$ and $\mathcal{S}^{\text {as }}$, scattering equations are directly obtained as effective equations in spectator degrees of freedom.

The plan of this paper is as follows. After establishing in Sec. II A the notation and the general features of the three-particle system, pseudostates are discussed in II B. The construction of the asymptotic channel spaces and the characterization of the asymptotic relative-motion (spectator) space are given in IIC and $\mathrm{II} \mathrm{D}$, respectively. In $\mathrm{Sec}$. II $\mathrm{E}$, the correspondence between the full approximation space and the asymptotic spectator space is setup. The formulation of the effec- tive wave-function equations on the asymptotic relativemotion space is given in Sec. III A. Lippmann-Schwingertype integral equations for the (effective) transition operators are presented in Sec. III B, followed by the derivation of alternative sets of transition-operator equations in Sec. III C. Computational procedures and numerical results are presented in Sec. IV. Finally, in Sec. V, concluding remarks are made.

\section{SPECIFICATION OF THE APPROXIMATION SPACE}

\section{A. Kinematics and notation}

The standard three-particle notation [2] and Jacobi variables will be used throughout this paper. With $(\alpha \beta \gamma)$ standing for cyclic permutations of (123), a two-fragment partition $(\alpha)(\beta \gamma)$ of the three particles is referred to as the rearrangement channel $\alpha$. The label $\alpha$ of the spectator particle in the partition $(\alpha)(\beta \gamma)$ is also used as a label for the pair $(\beta \gamma)$. The partition $(1)(2)(3)$ is referred to as the breakup channel. The Jacobi coordinates for the arrangement $\alpha$ are denoted by $\mathbf{x}_{\alpha}$ and $\mathbf{y}_{\alpha}$, with the corresponding reduced masses being $\mu_{\alpha}$ and $\nu_{\alpha}$, respectively. Here $\mathbf{x}_{\alpha}$ is the internal relative coordinate of the pair $(\beta \gamma)$, and $\mathbf{y}_{\alpha}$ the relative position of the spectator particle $\alpha$ with respect to the center of mass of the pair $(\beta \gamma)$. The momenta conjugate to $\mathbf{x}_{\alpha}$ and $\mathbf{y}_{\alpha}$ are $\mathbf{p}_{\alpha}$ and $\mathbf{q}_{\alpha}$, respectively.

The total barycentric Hamiltonian $H$ of the system is given as $H=H_{0}+V$. Here, $H_{0}$ is the kinetic energy operator, and $V$ the total interaction. $H_{0}$ can be decomposed as $H_{0}=k_{\alpha}+K^{\alpha}$, where $k_{\alpha}=p_{\alpha}^{2} /\left(2 \mu_{\alpha}\right)$, and $K^{\alpha}=$ $q_{\alpha}^{2} /\left(2 \nu_{\alpha}\right), \alpha=1,2,3$. Its eigenstates are the plane-wave states $\left|\mathbf{p}_{\alpha} \mathbf{q}_{\alpha}\right\rangle$.

The internal Hamiltonian for the pair $(\beta \gamma)$ is $h_{\alpha}=k_{\alpha}+$ $V_{\alpha}$, where $V_{\alpha}$ is the potential between particles $\beta$ and $\gamma$. Bound states of $\mathrm{h}_{\alpha}$ are denoted $\left|\varphi_{\alpha n}\right\rangle, \quad n=1, \ldots, N_{\alpha \mathrm{B}}$, with energies $\epsilon_{\alpha n}$, where $n$ stands for the collection of quantum numbers for the bound states. The scattering states will be denoted by $\left|\varphi_{\alpha \mathbf{p}}^{( \pm)}\right\rangle$

The rearrangement-channel Hamiltonian $H_{\alpha}$ (三 $\left.K^{\alpha}+h_{\alpha}\right)$ have the eigenkets $\left|\varphi_{\alpha n} \mathbf{q}_{\alpha}\right\rangle$ with energies $E_{\alpha n q}=\epsilon_{\alpha n}+q_{\alpha}^{2} /\left(2 \nu_{\alpha}\right)$. The channel interactions $V^{\alpha}$ are defined by $V^{\alpha}=V-V_{\alpha}$.

The post-form transition operators $[2,22] U_{\beta \alpha}^{(+)}(z)$ are defined as

$$
U_{\beta \alpha}^{(+)}(z)=V^{\beta} G(z) G_{\alpha}(z)^{-1}=V^{\beta}+V^{\beta} G(z) V^{\alpha},
$$

where $G(z)=(z-H)^{-1}$, and $G_{\alpha}(z)=\left(z-H_{\alpha}\right)^{-1}$ Here $z$ is a complex energy parameter. For physical scattering, $z=E+i \eta$, with the limit $\eta \rightarrow 0^{+}$being implicit. For a collision starting in channel $(\alpha n)$ with the initial state $\left|\phi_{\alpha n} \overline{\mathbf{q}}_{\alpha n}\right\rangle$, the amplitude for the transition $(\alpha n) \rightarrow\left(\beta n^{\prime}\right)$ is given by the onshell matrix element $\left\langle\phi_{\beta n^{\prime}} \overline{\mathbf{q}}_{\beta n^{\prime}}\left|\mathrm{U}_{\beta \alpha}^{(+)}(E)\right| \phi_{\alpha n} \overline{\mathbf{q}}_{\alpha n}\right\rangle$, where $E=$ $e_{\alpha n}+\bar{q}_{\alpha n}^{2} / 2 \nu_{\alpha}=e_{\beta n^{\prime}}+\bar{q}_{\beta n^{\prime}}^{2} 2 \nu_{\beta}$. Note that a bar over a momentum variable will be used to distinguish the 
on-shell value of the momentum from arbitrary (off-shell) values.

\section{B. Approximation subspaces for two-particle subsystems}

The channel structure of the approximate scattering theory is specified by choosing for every two-particle subsystem $\alpha$ a finite $L^{2}$ subspace $s_{\alpha}$ of dimension $N_{\alpha}$. Denoting with $\pi_{\alpha}$ the projector onto $s_{\alpha}$, the approximation scheme entails the replacement of the pair Hamiltonian $h_{\alpha}$ by $h_{\alpha \pi}\left(\equiv \pi_{\alpha} h_{\alpha} \pi_{\alpha}\right)$.

To construct $s_{\alpha}$, we first choose a primitive space $\tilde{s}_{\alpha}$, spanned by a suitable and sufficiently large set of orthonormal $L^{2}$ basis states $\left|u_{\alpha n}\right\rangle, n=1,2, \ldots, N_{\alpha, \max }$ Then, the pair Hamiltonian $h_{\alpha}$ is diagonalized in $\tilde{s}_{\alpha}$

$$
\sum_{n^{\prime \prime}=1}^{N_{\alpha, \max }}\left\langle u_{\alpha n^{\prime}}\left|h_{\alpha}\right| u_{\alpha n^{\prime \prime}}\right\rangle c_{\alpha, n^{\prime \prime} n}=c_{\alpha, n^{\prime} n} e_{\alpha n}
$$

where $n, n^{\prime}=1,2, \ldots, N_{\alpha, \max }$. The states defined as $\left|\phi_{\alpha n}\right\rangle=\sum_{n_{\alpha, \max }^{\prime}}^{N_{\alpha=1}}\left|u_{\alpha n^{\prime}}\right\rangle c_{\alpha, n^{\prime} n}$ are the eigenstates of the restriction of $h_{\alpha}$ on $\tilde{s}_{\alpha}$, with corresponding eigenenergies $\mathrm{e}_{\alpha n}$. For the approximate theory to possess the correct thresholds for the physical reaction channels, we assume that $\tilde{s}_{\alpha}$ has been chosen so that $\left|\phi_{\alpha n}\right\rangle, n=1,2, \ldots, N_{\alpha \mathrm{B}}$ are good approximations to the bound states $\left|\varphi_{\alpha n}\right\rangle$. The remaining states $\left|\phi_{\alpha n}\right\rangle, n=N_{\alpha_{\mathrm{B}}}+1, \ldots, N_{\alpha, \max }$, have positive energies and are referred to as the pseudostates embedded in the continuum.

Clearly, the pseudostates do not represent an invariant property of the pair $\alpha$. In particular, the spectrum of pseudostate energies can be varied by changing the primitive space $\tilde{s}_{\alpha}$. Nevertheless, a given set of pseudostates carry information about the continuum of the two-particle system. In particular, it is well known $[8,22]$ that a pseudostate $\left|\phi_{\alpha n}\right\rangle$ is an approximation (apart from a renormalization factor $w_{\alpha n}$ ) to $\varphi_{\alpha p_{n}}^{(-)}$, with $p_{n}=\sqrt{2 \mu_{\alpha} e_{\alpha n}}$. That is, a set of pseudostates corresponds, in a distributional sense, to a quadrature discretization of the continuous spectrum of the pair $\alpha$, with $\left\{e_{\alpha n}\right\}$ and $\left\{w_{\alpha n}\right\}$ being, respectively, the abscissa and the weights of the quadrature rule.

Assuming that the states $\left|\phi_{\alpha n}\right\rangle$ are enumerated in increasing order of energy, the subspace spanned by the lowest $N_{\alpha}$ states is taken as the approximation subspace $s_{\alpha}$ for the two-particle subsystem $\alpha$. Note that this subspace includes all of the bound states and some (or all) of the pseudostates, i.e., $N_{\alpha \mathrm{B}}<N_{\alpha} \leq N_{\alpha, \max }$ A sequence of approximate theories can be generated by varying $N_{\alpha}$. The pseudostates included in the approximate theory define pseudoreaction channels which simulate the breakup channel of the exact scattering system. The one-dimensional subspace (of $s_{\alpha}$ ) spanned by $\left|\phi_{\alpha n}\right\rangle$ will be denoted by $s_{\alpha n}$, and the corresponding projector by $\pi_{\alpha n}\left(\equiv\left|\phi_{\alpha n}\right\rangle\left\langle\phi_{\alpha n}\right|\right)$. We can then write $\pi_{\alpha}=\sum_{n=1}^{N_{\alpha}} \pi_{\alpha n}$ and $h_{\alpha \pi}=\sum_{n=1}^{N_{\alpha}} e_{\alpha n} \pi_{\alpha n}$.

\section{Approximation subspaces for rearrangement channels}

For each two-particle subspace $s_{\alpha n}$, we specify a corresponding spectator space $\mathcal{S}^{\alpha n}$, whose projector is denoted by $\Pi^{\alpha n}$. At this stage, we do not introduce any approximations with respect to the spectator degrees of freedom. Thus, each $\mathcal{S}^{\alpha n}$ represents the full state space for the spectator particle $\alpha$. That is, $\Pi_{\alpha n}=I^{\alpha}$, with $I^{\alpha}$ denoting the unit operator for the relative motion of the spectator particle $\alpha$, viz., $I^{\alpha}=\int d \mathbf{q}_{\alpha}\left|\mathbf{q}_{\alpha}\right\rangle\left\langle\mathbf{q}_{\alpha}\right|=$ $\int d \mathbf{y}_{\alpha}\left|\mathbf{y}_{\alpha}\right\rangle\left\langle\mathbf{y}_{\alpha}\right|$. To describe the asymptotic free motion of the spectator particle $\alpha$ in channel $(\alpha n)$, an effective free Hamiltonian $\mathcal{H}_{0}^{\alpha n}$ is defined on $\mathcal{S}^{\alpha n}$ via $\mathcal{H}_{0}^{\alpha n}=e_{\alpha n} \Pi^{\alpha n}+K^{\alpha}$. Operators acting only on spectator degrees of freedom will be distinguished by script symbols.

The direct product $s_{\alpha n} \otimes \mathcal{S}^{\alpha n}$ defines the channel subspace $\mathcal{S}_{\alpha n}$, with the correponding projector being $\Pi_{\alpha n}=$ $\pi_{\alpha n} \otimes I^{\alpha n}$. The asymptotic dynamics in channel $(\alpha n)$ is described by $H_{\alpha n}=\Pi_{\alpha n} H_{\alpha} \Pi_{\alpha n}=\pi_{\alpha n} \otimes \mathcal{H}_{0}^{\alpha n}$. The approximation subspace $\mathcal{S}_{\alpha}$ for the $\alpha$ th rearrangement is then defined as the (internal) direct sum $\oplus_{n=1}^{N_{\alpha}} \mathcal{S}_{\alpha n}$. The projector onto $\mathcal{S}_{\alpha}$ is given as $\Pi_{\alpha}=\sum_{n=1}^{N_{\alpha}} \Pi_{\alpha n}$, and $H_{\alpha}$ is replaced in the approximate theory by $H_{\alpha \pi}=\Pi_{\alpha} H_{\alpha} \Pi_{\alpha}$ $=\sum_{n=1}^{N_{\alpha}} \pi_{\alpha n} \otimes \mathcal{H}_{0}^{\alpha n}$.

\section{Asymptotic spectator space}

We define the asymptotic spectator space $\mathcal{S}^{\text {as }}$ as the (external) direct-sum space $\oplus_{\alpha n} \mathcal{S}^{\alpha n}$. A practical realization of this (external) direct-sum space is obtained by adopting a definite ordering of channels $(\alpha n)$, and representing a ket $|\mathbf{f}\rangle \in \mathcal{S}^{\text {as }}$ with the $(N \times 1)$ column vector of component kets $\left|f^{\alpha n}\right\rangle \in \mathcal{S}^{\alpha n}, n=1,2, \ldots, N_{\alpha}, \alpha=1,2,3$. Here $N=N_{1}+N_{2}+N_{3}$. Similarly, a bra $\langle\mathbf{f}|$ stands for a $(1 \times N)$ row vector of components $\left\langle f^{\alpha n}\right|$. The projector onto $\mathcal{S}^{\text {as }}$ is represented by a diagonal matrix $\Pi^{\text {as }}$ of projectors $\Pi^{\alpha n}$

We next introduce the overlap operators $\Delta^{\alpha n, \beta n^{\prime}}$ : $\mathcal{S}^{\beta n^{\prime}} \rightarrow \mathcal{S}^{\alpha n}$ by

$$
\begin{aligned}
\Delta^{\alpha n, \beta n^{\prime}} & =\int d \mathbf{q}_{\alpha} \int d \mathbf{q}_{\beta}\left|\mathbf{q}_{\alpha}\right\rangle \Delta^{\alpha n, \beta n^{\prime}}\left(\mathbf{q}_{\alpha}, \mathbf{q}_{\beta}\right)\left\langle\mathbf{q}_{\beta}\right| \\
& =\int d \mathbf{y}_{\alpha} \int d \mathbf{y}_{\beta}\left|\mathbf{y}_{\alpha}\right\rangle \Delta^{\alpha n, \beta n^{\prime}}\left(\mathbf{y}_{\alpha}, \mathbf{y}_{\beta}\right)\left\langle\mathbf{y}_{\beta}\right|
\end{aligned}
$$

where $\Delta^{\alpha n, \beta n^{\prime}}\left(\mathbf{q}_{\alpha}, \mathbf{q}_{\beta}\right)=\left\langle\phi_{\alpha n} \mathbf{q}_{\alpha} \mid \phi_{\beta n^{\prime}} \mathbf{q}_{\beta}\right\rangle$, and $\Delta^{\alpha n, \beta n^{\prime}}\left(\mathbf{y}_{\alpha}, \mathbf{y}_{\beta}\right)=\left\langle\phi_{\alpha n} \mathbf{y}_{\alpha} \mid \phi_{\beta n^{\prime}} \mathbf{y}_{\beta}\right\rangle$. Note that , $\Delta^{\alpha n, \beta n^{\prime}}\left(\mathbf{y}_{\alpha}, \mathbf{y}_{\beta}\right) \rightarrow \delta_{\alpha \beta} \delta_{n n^{\prime}} \delta\left(\mathbf{y}_{\alpha}-\mathbf{y}_{\beta}\right) \quad$ as $y_{\alpha}$ (or $\left.y_{\beta}\right) \rightarrow \infty$. That is, channel orthogonality holds only asymptotically.

We collect $\left\{\Delta^{\alpha n, \beta n^{\prime}}\right\}$ in the matrix $\Delta$, and define the nonorthogonality operators $\mathcal{N} \equiv \Delta-\boldsymbol{\Pi}^{\text {as }}$. That is, $\mathcal{N}^{\alpha n, \beta n^{\prime}}=\bar{\delta}_{\alpha \beta} \Delta^{\alpha n, \beta n^{\prime}}$, where $\bar{\delta}_{\alpha \beta}=1-\delta_{\alpha \beta}$. The inverse $\boldsymbol{\Lambda}$ of $\boldsymbol{\Delta}$ can now be defined via $\boldsymbol{\Delta} \boldsymbol{\Lambda}=\boldsymbol{\Pi}^{\text {as }}$, which can be rewritten as coupled integral equations for the kernels $\Lambda^{\alpha n, \beta n^{\prime}}\left(\mathbf{q}_{\alpha}, \mathbf{q}_{\beta}\right)$ : 


$$
\Lambda^{\alpha n, \beta n^{\prime}}\left(\mathbf{q}_{\alpha}, \mathbf{q}_{\beta}\right)=\delta_{\alpha \beta} \delta_{n n^{\prime}} \delta\left(\mathbf{q}_{\alpha}-\mathbf{q}_{\beta}\right)-\sum_{\gamma=1}^{3} \sum_{n^{\prime \prime}=1}^{N_{\gamma}} \int d \mathbf{q}_{\gamma} \mathcal{N}^{\alpha n, \gamma n^{\prime \prime}}\left(\mathbf{q}_{\alpha}, \mathbf{q}_{\gamma}\right) \Lambda^{\gamma n^{\prime \prime}, \beta n^{\prime}}\left(\mathbf{q}_{\gamma}, \mathbf{q}_{\beta}\right)
$$

A similar equation gives the coordinate-space kernel $\Lambda_{\alpha n, \beta n^{\prime}}\left(\mathbf{y}_{\alpha}, \mathbf{y}_{\beta}\right)$. The (formal or numerical) linear dependence problem would manifest itself as $\Delta$ becoming singular (formally or numerically), in which case $\boldsymbol{\Lambda}$ is to be considered as the generalized inverse of $\Delta$.

The aim of the theory is to express the collision dynamics in the form of effective (matrix) equations on $\mathcal{S}^{\text {as }}$. Towards this end, we collect the free spectator Hamiltonians $\mathcal{H}_{0}^{\alpha n}$ in the diagonal matrix $\mathcal{H}_{0}$, and define the effective matrix operators $\mathcal{H}, \mathcal{V}^{( \pm)}$, and $\mathcal{Z}^{( \pm)}$by

$$
\begin{aligned}
& \mathcal{H}^{\alpha n, \beta n^{\prime}}\left(\mathbf{q}_{\alpha}, \mathbf{q}_{\beta}\right)=\left\langle\phi_{\alpha n} \mathbf{q}_{\alpha}|H| \phi_{\beta n^{\prime}} \mathbf{q}_{\beta}\right\rangle, \\
& \mathcal{V}^{(+) \alpha n, \beta n^{\prime}}\left(\mathbf{q}_{\alpha}, \mathbf{q}_{\beta}\right)=\left\langle\phi_{\alpha n} \mathbf{q}_{\alpha}\left|V^{\alpha}\right| \phi_{\beta n^{\prime}} \mathbf{q}_{\beta}\right\rangle, \\
& \mathcal{V}^{(-) \alpha n, \beta n^{\prime}}\left(\mathbf{q}_{\alpha}, \mathbf{q}_{\beta}\right)=\left\langle\phi_{\alpha n} \mathbf{q}_{\alpha}\left|V^{\beta}\right| \phi_{\beta n^{\prime}} \mathbf{q}_{\beta}\right\rangle, \\
& \mathcal{Z}^{(+) \alpha n, \beta n^{\prime}}\left(\mathbf{q}_{\alpha}, \mathbf{q}_{\beta}\right)=\bar{\delta}_{\alpha \beta}\left\langle\phi_{\alpha n} \mathbf{q}_{\alpha}\left|H_{\alpha}\right| \phi_{\beta n^{\prime}} \mathbf{q}_{\beta}\right\rangle, \\
& \mathcal{Z}^{(-) \alpha n, \beta n^{\prime}}\left(\mathbf{q}_{\alpha}, \mathbf{q}_{\beta}\right)=\bar{\delta}_{\alpha \beta}\left\langle\phi_{\alpha n} \mathbf{q}_{\alpha}\left|H_{\beta}\right| \phi_{\beta n^{\prime}} \mathbf{q}_{\beta}\right\rangle,
\end{aligned}
$$

Coordinate-space kernels of these operators are similarly defined. Note that, as $y_{\alpha}$ (or $y_{\beta}$ ) $\rightarrow \infty$, $\mathcal{V}^{( \pm) \alpha n, \beta n^{\prime}}\left(\mathbf{y}_{\alpha}, \mathbf{y}_{\beta}\right)$ and $\mathcal{Z}^{( \pm) \alpha n, \beta n^{\prime}}\left(\mathbf{y}_{\alpha}, \mathbf{y}_{\beta}\right) \rightarrow 0$. That is, $\mathcal{H}$ asymptotically approaches $\mathcal{H}_{0}$, and is decomposable as $\mathcal{H}=\mathcal{H}_{0}+\mathcal{V}^{( \pm)}+\mathcal{Z}^{( \pm)}$.

\section{E. The full approximation space}

The full approximation space $\mathcal{S}$ is taken as the linear spari of three rearrangement subspaces $\mathcal{S}_{1}, \mathcal{S}_{2}$, and $\mathcal{S}_{3}$. Due to the nonorthogonality of the subspaces for two distinct rearrangements (i.e, $\Pi_{\alpha} \Pi_{\beta} \neq \delta_{\alpha \beta} \Pi_{\alpha}$ for $\alpha \neq \beta$ ), the characterization of the space $\mathcal{S}$, and the construction of the corresponding projector $\mathrm{P}_{\pi}$, is somewhat involved, especially, when there is linear dependence between rearrangement subspaces.

Let us now consider a state $|\Psi\rangle \in \mathcal{S}$, and define $\left|\bar{f}^{\alpha n}\right\rangle=\left\langle\phi_{\alpha n} \mid \Psi\right\rangle$, where the inner product is over only the internal variables of the pair $\alpha$, i.e., $\bar{f}^{\alpha n}\left(\mathbf{q}_{\alpha}\right)=\left\langle\phi_{\alpha n} \mathbf{q}_{\alpha} \mid \Psi\right\rangle$. We collect $\left|\bar{f}^{\alpha n}\right\rangle \in \mathcal{S}^{\alpha n}, n=$ $1,2, . ., N_{\alpha}, \alpha=1,2,3$, in the column vector $|\overline{\mathbf{f}}\rangle \in \mathcal{S}^{\text {as }}$, and define $|\mathbf{f}\rangle=\boldsymbol{\Lambda}|\overline{\mathbf{f}}\rangle$. Any state $|\Psi\rangle \in \mathcal{S}$ then has the decomposition $|\Psi\rangle=\sum_{\alpha n}\left|\phi_{\alpha n} f^{\alpha n}\right\rangle$. The states $|\mathbf{f}\rangle$ and $|\overline{\mathbf{f}}\rangle$ form a biorthogonal pair in $\mathcal{S}^{\text {as }}$.

To set up the communication between $\mathcal{S}^{\text {as }}$ and $\mathcal{S}$, we first define the channel injection operators $[16,17$, 20] operating between $\mathcal{S}_{\alpha n}$ and $\mathcal{S}^{\alpha n}: \mathcal{J}_{\alpha n}\left|f^{\alpha n}\right\rangle=$ $\left|\phi_{\alpha n} f^{\alpha n}\right\rangle$, and $\mathcal{J}_{\alpha n}^{*}|\Psi\rangle=\left|\bar{f}^{\alpha n}\right\rangle$. With these definitions, $\mathcal{J}_{\alpha n} \mathcal{J}_{\alpha n^{\prime}}^{*}=\delta_{n n^{\prime}} \Pi_{\alpha n}$ and $\mathcal{J}_{\alpha n}^{*} \mathcal{J}_{\alpha n^{\prime}}=\delta_{n n^{\prime}} \Pi^{\alpha n}$. More generally, $\mathcal{J}_{\alpha n} \mathcal{J}_{\beta n^{\prime}}^{*}=\Pi_{\alpha n} \Pi_{\beta n^{\prime}}$, and $\mathcal{J}_{\alpha n}^{*} \mathcal{J}_{\beta n^{\prime}}=\Delta^{\alpha n, \beta n^{\prime}}$. The communication between $\mathcal{S}$ and $\mathcal{S}^{\text {as }}$ will be provided by the injection operators $\mathcal{J}: \mathcal{S}^{\text {as }} \rightarrow \mathcal{S}$ and $\mathcal{J}^{*}$ : $\mathcal{S}^{\text {as }} \rightarrow \mathcal{S}$. We take $\mathcal{J}$ as the $(1 \times N)$ row vector with components $\mathcal{J}_{\alpha n}$, and $\mathcal{J}^{*}$ as the $(N \times 1)$ column vector with components $\mathcal{J}_{\alpha n}^{*}, \quad n=1,2 \ldots, N_{\alpha}, \alpha=1,2,3$. With these definitions, the correpondence between $\mathcal{S}$ and $\mathcal{S}^{\text {as }}$ can now be written as $\mathcal{J}|\mathbf{f}\rangle=\sum_{\alpha=1}^{3} \sum_{n=1}^{N_{\alpha}}\left|\phi_{\alpha n} f^{\alpha n}\right\rangle$ $=|\Psi\rangle$, and $\mathcal{J}^{*}|\Psi\rangle=|\overline{\mathbf{f}}\rangle=\boldsymbol{\Delta}|\mathbf{f}\rangle$. Other choices of the injection operators have been considered by Kouri and Hoffmann [24].

It follows from these definitions that $\mathcal{J}^{*} \mathcal{J}=\boldsymbol{\Delta}$, and $\mathcal{J J}^{*}=\sum_{\alpha=1}^{3} \sum_{n=1}^{N_{\alpha}} \Pi_{\alpha n}$. If we instead use as injection operators the pair $\left(\mathcal{J}, \mathcal{J}^{\prime *}\right)$, where $\mathcal{J}^{* *}=\boldsymbol{\Lambda} \mathcal{J}^{*}$, we obtain $\Pi^{\text {as }}=\mathcal{J}^{\prime *} \mathcal{J}$, and $P_{\pi}=\mathcal{J} \mathcal{J}^{\prime *}$. That is, $P_{\pi}=\mathcal{J} \boldsymbol{\Lambda}$ $\mathcal{J}^{*}$, or, in explicit notation,

$$
P_{\pi}=\sum_{\alpha=1}^{3} \sum_{n=1}^{N_{\alpha}} \sum_{\beta=1}^{3} \sum_{n^{\prime}=1}^{N_{\beta}} \int d \mathbf{q}_{\alpha} \int d \mathbf{q}_{\beta}\left|\phi_{\alpha n} \mathbf{q}_{\alpha}\right\rangle \Lambda_{\alpha n, \beta n^{\prime}}\left(\mathbf{q}_{\alpha}, \mathbf{q}_{\beta}\right)\left\langle\phi_{\beta n^{\prime}} \mathbf{q}_{\beta}\right|
$$

In passing, we note that the spectator-space analog of $\mathcal{J} \mathcal{J}^{*}$ is $\Delta^{2}$. Hence, $\left(\mathcal{J} \mathcal{J}^{*}\right)^{-1}=\mathcal{J} \Delta^{-2} \mathcal{J}^{*}$. This yields the alternative expression $[16,20]: P_{\pi}=\left(\mathcal{J J}^{*}\right)^{-1} \mathcal{J J}^{*}$. We can similarly show that $\mathrm{P}_{\pi} \mathcal{J}=\mathcal{J}$, and $\mathcal{J}^{*} \mathrm{P}_{\pi}=$ $\mathcal{J}^{*}$, and $\mathcal{J}^{*} \mathrm{P}_{\pi} \mathcal{J}=\Delta$. It is also easy to derive the $\mathcal{S}^{\text {as }}$-space counterparts of these relationships: $\Pi^{\text {as }}=$ $\mathcal{J}^{*}\left(\mathcal{J} \mathcal{J}^{*}\right)^{-1} \mathcal{J}=\left(\mathcal{J}^{*} \mathcal{J}\right)^{-1} \mathcal{J}^{*} \mathcal{J}, \mathcal{J} \Pi^{\text {as }}=\mathcal{J}$, and $\Pi^{\text {as }} \mathcal{J}^{*}=\mathcal{J}^{*}$.

In going back and forth between the spaces $\mathcal{S}$ and $\mathcal{S}^{\text {as }}$, operators are transformed according to $\mathcal{O}=\mathcal{J}^{*} O \mathcal{J}$, and $O=\mathcal{J} \boldsymbol{\Lambda} \mathcal{O} \Lambda \mathcal{J}^{*}$. Defining the inverse operators $O^{-1}$ and $\mathcal{O}^{-1}$ via $O O^{-1}=P_{\pi}$, and $\mathcal{O} \mathcal{O}^{-1}=\Pi^{\text {as }}$, respectively, we can write $O^{-1}=\mathcal{J O}^{-1} \mathcal{J}^{*}$.

The restriction $H_{\pi}\left(=P_{\pi} H P_{\pi}\right)$ of $H$ on $\mathcal{S}$ will play the role of the total Hamiltonian on the scattering theory to be developed on $\mathcal{S}$. Note that $\mathcal{J}^{*} H \mathcal{J}=\mathcal{H}$, and

$$
H_{\pi}=P_{\pi} H P_{\pi}=\mathcal{J} \mathbf{\Lambda} \mathcal{H} \mathbf{\Lambda} \mathcal{J}^{*} .
$$

\section{SCATTERING THEORY ON THE ASYMPTOTIC SPECTATOR SPACE}

\section{A. Wave-function equations}

We consider a collision process in which particle $\alpha$ with initial relative momentum $\overline{\mathbf{q}}_{\alpha n}$ is incident on the bound pair $(\beta \gamma)$, initially in state state $\left|\phi_{\alpha n}\right\rangle$. Thus, the total energy is $E=e_{\alpha n}+\bar{q}_{\alpha n}^{2} /\left(2 \nu_{\alpha}\right)$. The initial channel state $\left|\phi_{\alpha n} \overline{\mathbf{q}}_{\alpha n}\right\rangle$ will be represented in $\mathcal{S}^{\text {as }}$ by $\left|\mathbf{i}_{\alpha n \overline{\mathbf{q}}}\right\rangle$, i.e., 
$\left|\phi_{\alpha n} \overline{\mathbf{q}}_{\alpha n}\right\rangle=\mathcal{J}\left|\mathbf{i}_{\alpha n \overline{\mathbf{q}}}\right\rangle$. Here, $\left|\mathbf{i}_{\alpha n \overline{\mathbf{q}}}\right\rangle$ denotes a column vector with components $\left|i_{\alpha n \overline{\mathbf{q}}}^{\beta n^{\prime}}\right\rangle=\delta_{\alpha \beta} \delta_{n n^{\prime}}\left|\overline{\mathbf{q}}_{\alpha n}\right\rangle$. Obviously, $\left|\mathbf{i}_{\alpha n \overline{\mathbf{q}}}\right\rangle$ is an (improper) eigenstate of $\mathcal{H}_{0}$ with energy $E=\mathrm{e}_{\alpha n}+\bar{q}_{\alpha n}^{2} / 2 \nu_{\alpha}$.

The exact Schrodinger equation of the collision system is replaced on $\mathcal{S}$ by $\mathrm{H}_{\pi}\left|\Psi_{\alpha n \overline{\mathbf{q}}}\right\rangle=E\left|\Psi_{\alpha n \overline{\mathbf{q}}}\right\rangle$, where $\left|\Psi_{\alpha n \bar{q}}\right\rangle \in \mathcal{S}$. By defining $\left\langle\mathbf{q}_{\beta} \mid \bar{f}_{\alpha n \bar{q}}^{\beta n^{\prime}}\right\rangle=\left\langle\phi_{\beta n} \mathbf{q}_{\beta} \mid \Psi_{\alpha n \bar{q}}\right\rangle$, and $\left|f_{\alpha n \bar{q}}^{\beta n^{\prime}}\right\rangle=\sum_{\gamma=1}^{3} \sum_{n^{\prime \prime}=1}^{N_{\gamma}} \Delta^{\beta n^{\prime}, \gamma n^{\prime \prime}}\left|\bar{f}_{\alpha n \bar{q}}^{\gamma n^{\prime \prime}}\right\rangle$, the approximate scattering state $\left|\Psi_{\alpha n \bar{q}}\right\rangle$ can be written as $\left|\Psi_{\alpha n \bar{q}}\right\rangle=\sum_{\beta, n^{\prime}}\left|\phi_{\beta n^{\prime}} f_{\alpha n \bar{q}}^{\beta n^{\prime}}\right\rangle$. Collecting $\left\{\left|f_{\alpha n \bar{q}}^{\beta n^{\prime}}\right\rangle\right\}$ and $\left\{\left|\bar{f}_{\alpha n \bar{q}}^{\beta n^{\prime}}\right\rangle\right\}$ in column vectors $\left|\mathbf{f}_{\alpha n \bar{q}}\right\rangle$ and $\left|\overline{\mathbf{f}}_{\alpha n \bar{q}}\right\rangle$, respectively, we have $\mathcal{J}^{*}\left|\Psi_{\alpha n \bar{q}}\right\rangle=\left|\overline{\mathbf{f}}_{\alpha n \bar{q}}\right\rangle$ and $\mathcal{J}\left|\mathbf{f}_{\alpha n \bar{q}}\right\rangle=\left|\Psi_{\alpha n \bar{q}}\right\rangle$. Using $H_{\pi}=\mathcal{J} \mathbf{\Lambda} \mathcal{H} \boldsymbol{\Lambda} \mathcal{J}^{*}$ and $P_{\pi}=\mathcal{J} \boldsymbol{\Lambda} \mathcal{J}^{*}$, the $\mathcal{S}$-space Schrödinger equation becomes $\mathcal{J} \boldsymbol{\Lambda}(\mathcal{H}-E \Delta)|\mathbf{f}\rangle=0$. Multiplying from left with $\mathcal{J}^{*}$, and using $\mathcal{J}^{*} \mathcal{J}=\boldsymbol{\Delta}$, we obtain the effective Schrödinger equation on $\mathcal{S}^{\text {as }}$ :

$$
(E \Delta-\mathcal{H})|\mathbf{f}\rangle=0
$$

which reads in explicit notation

$$
\begin{aligned}
& \left(E-\mathrm{e}_{\beta n^{\prime}}-\frac{q_{\beta}^{2}}{2 \nu_{\beta}}\right) f_{\alpha n \bar{q}}^{\beta n^{\prime}}\left(\mathbf{q}_{\beta}\right) \\
& =\sum_{\gamma, n^{\prime \prime}} \int d \mathbf{q}_{\gamma} \mathcal{V}^{\beta n^{\prime}, \gamma n^{\prime \prime}}\left(\mathbf{q}_{\beta}, \mathbf{q}_{\gamma} ; E\right) f_{\alpha n \bar{q}}^{\gamma n^{\prime \prime}}\left(\mathbf{q}_{\gamma}\right)
\end{aligned}
$$

where

$$
\begin{aligned}
& \mathcal{V}^{\beta n^{\prime}, \alpha n}(E)=\mathcal{V}^{( \pm) \beta n^{\prime}, \alpha n}+\mathcal{W}^{( \pm) \beta n^{\prime}, \alpha n}(E) \\
& \mathcal{W}^{( \pm) \beta n^{\prime}, \alpha n}(E)=\mathcal{Z}^{( \pm) \beta n^{\prime}, \alpha n}-E \mathcal{N}^{\beta n^{\prime}, \alpha n} .
\end{aligned}
$$

Coordinate-space version of this equation is a coupled set of integrodifferential equations for $f_{\alpha n \bar{q}}^{\beta n^{\prime}}\left(\mathbf{y}_{\beta}\right)$. By construction the space $\mathcal{S}$ can accommodate outgoing waves in only the spectator coordinates $\mathbf{y}_{\beta}, \beta=1,2,3$. Although this is sufficient to satisfy the rearrangementchannel boundary conditions, the breakup boundary conditions involving outgoing waves in hyperspherical radius cannot be fulfilled with functions belonging to $\mathcal{S}$, and have to be approximated. In accordance with the nature of the approximation space, for all channels $\left(\beta n^{\prime}\right)$ with $E>e_{\beta n^{\prime}}$, the spectator functions $f_{\alpha n \overline{\mathbf{q}}}^{\beta n^{\prime}}$ will be required, as $y_{\beta} \rightarrow \infty$, to have the form

$$
f_{\alpha n \overline{\mathbf{q}}}^{\beta n^{\prime}}\left(\mathbf{y}_{\beta}\right) \rightarrow \delta_{\beta \alpha} \delta_{n^{\prime} n}(2 \pi)^{-3 / 2} e^{i \overline{\mathbf{q}}_{\alpha n} \cdot \mathbf{y}_{\alpha}}+\sqrt{2 \pi} \nu_{\beta} \mathcal{A}^{\beta n^{\prime}, \alpha n}\left(\bar{q}_{\beta n^{\prime}} \hat{y}_{\beta}, \overline{\mathbf{q}}_{\alpha n} ; E\right) e^{i \bar{q}_{\beta n^{\prime}} y_{\beta}} / y_{\beta}
$$

Here $\bar{q}_{\beta n^{\prime}}=\sqrt{2 \nu_{\beta}}\left(E-\mathrm{e}_{\beta n^{\prime}}\right)$, and $\mathcal{A}^{\beta n^{\prime}, \alpha n}\left(\overline{\mathbf{q}}_{\beta n}, \overline{\mathbf{q}}_{\alpha n} ; E\right)$ is, apart from a multiplicative constant, the scattering amplitude for the transition $(\alpha n) \rightarrow\left(\beta n^{\prime}\right)$.

Under these boundary conditions, the effective Schrodinger equation can be replaced by the integral equation

$$
\mathbf{f}_{\alpha n \overline{\mathbf{q}}}^{\beta n^{\prime}}\left(\mathbf{q}_{\beta}\right)=\delta_{\beta \alpha} \delta_{n n^{\prime}} \delta\left(\mathbf{q}_{\beta}-\overline{\mathbf{q}}_{\alpha n}\right)+\left(E+i \eta-\mathrm{e}_{\beta n^{\prime}}-{q^{\prime}}_{\beta}^{2} / 2 \nu_{\beta}\right)^{-1} \sum_{\gamma, n^{\prime \prime}} \int d \mathbf{q}_{\gamma} \mathcal{V}^{\beta n^{\prime}, \gamma n^{\prime \prime}}\left(\mathbf{q}_{\beta}, \mathbf{q}_{\gamma} ; E\right) f_{\alpha n \overline{\mathbf{q}}}^{\gamma n^{\prime \prime}}\left(\mathbf{q}_{\gamma}\right)
$$

which in matrix notation reads

$$
\left|\mathbf{f}_{\alpha n \overline{\mathbf{q}}}\right\rangle=\left|\mathbf{i}_{\alpha n \overline{\mathbf{q}}}\right\rangle+\mathcal{G}_{0}(E) \mathcal{V}(E)\left|\mathbf{f}_{\alpha n \overline{\mathbf{q}}}\right\rangle,
$$

where $\mathcal{G}_{0}(z)=\left(z \boldsymbol{\Pi}^{\text {as }}-\mathcal{H}_{0}\right)^{-1} \Pi^{\text {as }}$.

Introducing the wave operator $\Omega(E)$ via $\Omega(E)\left|\mathbf{i}_{\alpha n \overline{\mathbf{q}}}\right\rangle=$ $\left|\mathbf{f}_{\alpha n \overline{\mathbf{q}}}\right\rangle$, we can rewrite Eq.(13) in fully off-shell form as

$$
\Omega(z)=\Pi^{\text {as }}+\mathcal{G}_{0}(z) \mathcal{V}(z) \Omega(z) .
$$

Defining $\mathcal{G}(z)$ via

$$
(z \Delta-\mathcal{H}) \mathcal{G}=\Pi^{\text {as }},
$$

and using the resolvent relations $\mathcal{G}=\mathcal{G}_{0}+\mathcal{G}_{0} \mathcal{V G}=$ $\mathcal{G}_{0}+\mathcal{G} \mathcal{V G}_{0}$, the formal solution of Eq.(14) can be written as $\Omega=\mathcal{G G}_{0}^{-1}=\Pi^{\text {as }}+\mathcal{G} \mathcal{V}$.

Note that full resolvent $G_{\pi}$, defined by $\left(E P_{\pi}-\right.$ $\left.H_{\pi}\right) G_{\pi}=P_{\pi}$, is related to $\mathcal{G}$ by

$$
G_{\pi}=\mathcal{J G} \mathcal{J}^{*}=\mathcal{J}(E \Delta-\mathcal{H})^{-1} \mathcal{J}^{*}
$$

\section{B. Transition-operator equations}

Let us define the effective transition operators $\mathcal{A}^{\beta n^{\prime}, \alpha n}(E)$ via $\mathcal{A}(z)=\mathcal{V}(z) \boldsymbol{\Omega}(z)$. The integral equation for these transition operators follows from (14) as

$$
\mathcal{A}=\mathcal{V}+\mathcal{V} \mathcal{G}_{0} \mathcal{A}
$$

where we supressed the energy dependence of operators involved. From the asymptotic form of Eq.(12), we can now identify the amplitude $\mathcal{A}^{\beta n^{\prime}, \alpha n}\left(\overline{\mathbf{q}}_{\beta n}, \overline{\mathbf{q}}_{\alpha n} ; E\right)$ in (11) as the on-shell matrix element of $\mathcal{A}^{\beta n^{\prime}, \alpha n}(E)$. Putting Eq.(14) on the energy shell from the right, we obtain our basic set of integral equations :

$$
\mathcal{A}^{\beta n^{\prime}, \alpha n}\left(\mathbf{q}_{\beta}, \overline{\mathbf{q}}_{\alpha n} ; E\right)=\mathcal{V}^{\beta n^{\prime}, \alpha n}\left(\mathbf{q}_{\beta}, \overline{\mathbf{q}}_{\alpha n} ; E\right)+\sum_{\gamma, n^{\prime \prime}} \int d \mathbf{q}_{\gamma} \frac{\mathcal{V}^{\beta n^{\prime}, \gamma n^{\prime \prime}}\left(\mathbf{q}_{\beta}, \mathbf{q}_{\gamma} ; E\right) \mathcal{A}^{\gamma n^{\prime \prime}, \alpha n}\left(\mathbf{q}_{\gamma}, \overline{\mathbf{q}}_{\alpha} ; E\right)}{E+i \eta-e_{\gamma n^{\prime \prime}}-q_{\gamma}^{2} / 2 \nu_{\gamma}}
$$


We note that $\mathcal{A}$ satisfy also the left-hand form of Eq.(17), viz., $\mathcal{A}=\mathcal{V}+\mathcal{A} \mathcal{G}_{0} \mathcal{V}$, as well as all the relationships familiar from the two-particle scattering theory, e.g., $\mathcal{A}=\mathcal{V} \mathcal{G G}_{0}^{-1}=\mathcal{G}_{0}^{-1} \mathcal{G} \mathcal{V}=\mathcal{V}+\mathcal{V} \mathcal{G} \mathcal{V}$, and

$$
\begin{aligned}
\mathcal{G} & =\mathcal{G}_{0}+\mathcal{G}_{0} \mathcal{A G}_{0} \\
\boldsymbol{\Omega} & =\boldsymbol{\Pi}^{\text {as }}+\mathcal{G}_{0} \mathcal{A} .
\end{aligned}
$$

These relationships manifestly show that the scattering theory on the approximation space finds its most symmetrical formulation in terms of the effective operator $\mathcal{A}$. However, if the solutions of the present approximate theory are to be used in context going beyond the approximation space $\mathcal{S}$, the connection of $\mathcal{A}^{\beta n^{\prime}, \alpha n}$ with the conventional transition operators of multichannel scattering theory have to be established. This can be achieved most easily by replacing $G$ in Eq.(1) with $G_{\pi}$, yielding an approximation to $U_{\beta \alpha}^{(+)}$:

$$
U_{\beta \alpha}^{(+) \pi}=V^{\beta} G_{\pi} G_{\alpha}^{-1}=V^{\beta} \mathcal{J G} \mathcal{J}^{*} G_{\alpha}^{-1} .
$$

Note that the domain and the range of $U_{\beta \alpha}^{(+) \pi}$ is not restricted to $\mathcal{S}$. Thus, Eq.(21) in conjunction with Eq.(19) can be used as an extrapolation formula to obtain approximations for matrix elements of $U_{\beta \alpha}^{(+)}$between states not contained in $\mathcal{S}$. For example, for a initial physical (rather than pseudo-) channel $(\alpha n)$, we find

$$
\left\langle\mathbf{p}_{\beta} \mathbf{q}_{\beta}\left|\mathrm{U}_{\alpha \beta}^{(+) \pi}(E)\right| \varphi_{\alpha n} \overline{\mathbf{q}}_{\alpha n}\right\rangle=\left\langle\mathbf{p}_{\beta} \mathbf{q}_{\beta}\left|\mathrm{V}^{\beta}\right| \varphi_{\alpha n} \overline{\mathbf{q}}_{\alpha n}\right\rangle+\sum_{\gamma, n^{\prime}} \int d \mathbf{q}_{\gamma} \frac{\left\langle\mathbf{p}_{\beta} \mathbf{q}_{\beta}\left|\mathrm{V}^{\beta}\right| \varphi_{\gamma n^{\prime}} \mathbf{q}_{\gamma}\right\rangle \mathcal{A}^{n^{\prime}, \alpha n}\left(\mathbf{q}_{\gamma}, \overline{\mathbf{q}}_{\alpha n} ; E\right)}{E+i \eta-e_{\gamma n^{\prime}}-q_{\gamma}^{2} / 2 \nu_{\gamma}} .
$$

In writing Eq. (22), we used $\mathcal{W}^{(-) \gamma n^{\prime}, \alpha n}\left(\mathbf{q}_{\gamma}, \overline{\mathbf{q}}_{\alpha n} ; E\right)=$ 0 , which is valid for $n=1,2, \ldots, N_{\alpha \mathrm{B}}$, with arbitrary $\gamma, n^{\prime}$ and $\mathbf{q}_{\gamma}$.

\section{Alternative integral equations}

Additional insight into the nature of the operator $\mathcal{A}$ can be obtained by introducing another transition operator $\mathcal{A}^{(+)}$via

$$
\mathcal{A}^{(+)}=\mathcal{V}^{(+)} \Omega \text {. }
$$

Using $\mathcal{V}=\mathcal{V}^{(+)}+\mathcal{W}^{(+)}$, we find $\mathcal{A}=\mathcal{A}^{(+)}+$ $\mathcal{W}^{(+)} \Omega$. Since $\left\langle\overline{\mathbf{q}}_{\beta n^{\prime}}\right| \mathcal{W}^{(+) \beta n^{\prime}, \alpha n}(E)=0$ for $n=1,2, \ldots, N_{\beta \mathrm{B}}, \beta=1,2,3$, the operators $\mathcal{A}$ and $\mathcal{A}^{(+)}$ have the same on-shell matrix elements as far as physical rearrangement channels are concerned. Note that $\left\langle\mathbf{q}_{\beta}\left|\mathcal{A}^{(+) \beta n^{\prime}, \alpha n}\right| \overline{\mathbf{q}}_{\alpha n}\right\rangle=\left\langle\phi_{\beta n^{\prime}} \mathbf{q}_{\beta}\left|\mathrm{V}^{\beta}\right| \Psi_{\alpha n \overline{\mathbf{q}}}\right\rangle$, which is just the integral formula for the transition $(\alpha n) \rightarrow\left(\beta n^{\prime}\right)$.

To derive the integral equation for $\mathcal{A}^{(+)}$, we first define the "nonorthogonality-distorted" channel resolvents $\mathcal{G}_{0}^{( \pm)}(z)=\left(z \boldsymbol{\Pi}^{\text {as }}-\mathcal{H}_{0}-\mathcal{W}^{( \pm)}\right)^{-1}$. Using the the resolvent relation $\mathcal{G}=\mathcal{G}_{0}^{( \pm)}+\mathcal{G}_{0}^{( \pm)} \mathcal{V}^{( \pm)} \mathcal{G}^{( \pm)}$, an alternate integral equation for $\boldsymbol{\Omega}$ follows:

$$
\boldsymbol{\Omega}=\mathcal{G}_{0}^{(+)} \mathcal{G}_{0}^{-1}+\mathcal{G}_{0}^{(+)} \mathcal{V}^{(+)} \boldsymbol{\Omega} \text {. }
$$

Since $\mathcal{A}^{(+)}=\mathcal{V}^{(+)} \boldsymbol{\Omega}$, we can immediately write down

$$
\mathcal{A}^{(+)}=\mathcal{V}^{(+)} \mathcal{G}_{0}^{(+)} \mathcal{G}_{0}^{-1}+\mathcal{V}^{(+)} \mathcal{G}_{0}^{(+)} \mathcal{A}^{(+)} \text {. }
$$

By further manipulation, this equation can be recast as

$$
\mathcal{A}^{(+)}=\mathcal{V}^{(+)}+\mathcal{A}^{(+)} \mathcal{G}_{0} \mathcal{V}
$$

This last equation represents, in the present context, the "post" version of the $M$ equation of Chandler and Gibson [20,21]. Note that it has the same kernel as the $\mathcal{A}$ equation, but a different inhomogeneous term. Consequently, the solution of Eq.(26) is related to that of Eq.(17) by $\mathcal{A}^{(+)}=\mathcal{A}-\mathcal{W}^{(+)}\left(\Pi^{\text {as }}+\mathcal{G}_{0} \mathcal{A}\right)$. This last relation is, in fact, equivalent to the integral formula $\mathcal{A}^{(+)}=\mathcal{V}^{(+)} \boldsymbol{\Omega}$, and can also be rewritten as

$$
\mathcal{A}^{(+)}=\left(\mathcal{G}_{0}^{(+)}\right)^{-1} \mathcal{G}_{0} \mathcal{A}-\mathcal{W}^{(+)},
$$

where $\left(\mathcal{G}_{0}^{(+)}\right)^{-1} \mathcal{G}_{0}=\boldsymbol{\Pi}^{\text {as }}-\mathcal{W}^{(+)} \mathcal{G}_{0}$. If the approximate theory involves just the physical reaction channels, but no pseudochannels, this relation becomes $\mathcal{A}^{(+)}=\mathcal{G}_{0}^{-1} \Delta \mathcal{G}_{0} \mathcal{A}-\mathcal{G}_{0}^{-1} \mathcal{N}$.

Although the effective operators $\mathcal{A}^{(+) \beta n^{\prime}, \alpha n}$ are certainly of the post type, they are not directly related to $U_{\alpha, \beta}^{(+)}$. The natural counterparts of $U_{\alpha, \beta}^{(+)}$in the approximate theory are the effective operators $T^{(+) \beta n^{\prime}, \alpha n}$, defined as

$$
\mathcal{T}^{(+) \beta n^{\prime}, \alpha n}=\mathcal{J}_{\beta n^{\prime}}^{*} U_{\beta \alpha}^{(+) \pi} \mathcal{J}_{\alpha n} .
$$

Noting that $\mathcal{J}_{\beta n^{\prime}}^{*}, \mathrm{~V}^{\beta} \mathcal{J}_{\gamma n^{\prime \prime}}=\mathcal{V}^{(+) \beta n^{\prime}, \gamma n^{\prime \prime}}$, and $\mathcal{J}_{\beta n^{\prime}}^{*}\left(E-\mathrm{H}_{\gamma}\right) \mathcal{J}_{\gamma n^{\prime \prime}} \quad$ is the $\left(\beta n^{\prime}, \gamma n^{\prime \prime}\right)$ th element of $\left(\mathcal{G}_{0}^{(+)}\right)^{-1}$, Eq. $(21)$ and $(28)$ can be combined to yield

$$
\mathcal{T}^{(+)}=\mathcal{V}^{(+)} \mathcal{G}^{(+)}\left(\mathcal{G}_{0}^{(-)}\right)^{-1}=\mathcal{V}^{(+)}+\mathcal{V}^{(+)} \mathcal{G} \mathcal{V}^{(-)}
$$

Using the resolvent relations, we can also write $\mathcal{G}=$ $\mathcal{G}_{0}^{(+)}+\mathcal{G}_{0}^{(+)} \mathcal{T}^{(+)} \mathcal{G}_{0}^{(-)}$. Thus these operators fully exhibit the asymmetry of $U_{\beta, \alpha}^{(+)}$with respect to the initial and final channel indices. Further manipulations yield the integral equations

$\mathcal{T}^{(+)}=\mathcal{V}^{(+)} \mathcal{G}_{0}^{(+)}\left(\mathcal{G}_{0}^{-}\right)^{-1}+\mathcal{V}^{(+)} \mathcal{G}_{0}^{(+)} \mathcal{T}^{(+)}$

$\mathcal{T}^{(+)}=\mathcal{V}^{(+)}+\boldsymbol{\Gamma}^{(+)} \mathcal{G}_{0}^{(-)} \mathcal{V}^{(-)}$

Comparing these equations with (25) and (26), we find that

$$
\mathcal{T}^{(+)}=\mathcal{A}^{(+)} \mathcal{G}_{0}\left(\mathcal{G}_{0}^{(-)}\right)^{-1} .
$$

Combining (27) and (32), the relationship between $\mathcal{T}^{(+)}$and $\mathcal{A}$ is established as 


$$
\begin{aligned}
\mathcal{T}^{(+)}= & \left(\mathcal{G}_{0}^{(+)}\right)^{-1} \mathcal{G}_{0} \mathcal{A} \mathcal{G}_{0}\left(\mathcal{G}_{0}^{(-)}\right)^{-1} \\
& -\mathcal{W}^{(+)} \mathcal{G}_{0}\left(\mathcal{G}_{0}^{(-)}\right)^{-1}
\end{aligned}
$$

For the special case of the approximate theory involving only the true reaction channels (thus ignoring breakup completely), we have

$$
\mathcal{T}^{(+)}=\mathcal{G}_{0}^{-1} \Delta \mathcal{A} \Delta \mathcal{G}_{0}^{-1}-\mathcal{G}_{0}^{-1} \mathcal{N} \mathcal{G}_{0} \Delta \mathcal{G}_{0}^{-1}
$$

Equation (33) shows that $\mathcal{T}^{(+)}$and $\mathcal{A}$ are equivalent as far as the on-shell matrix elements between physical rearrangement channels are concerned. The off-shell matrix elements of $\mathcal{T}^{(+)}$can be obtained from those $\mathcal{A}$ by quadrature, i.e, without having to directly solve $(30)$ or (31).

\section{COMPUTATIONAL IMPLEMENTATION AND RESULTS}

\section{A. Model}

The three-particle problems with finite-rank separable potentials can be numerically solved within the Faddeev formalism using techniques $[2,3]$ that are by now well established. In the simplest such model, the total interaction is pairwise additive, and the pair potentials are rank1 separable and restricted to act only on the $s$-wave states of the pairs, viz., $V=V_{1}+V_{2}+V_{3}$, and $V_{\alpha}=\left|\chi_{\alpha}\right\rangle \lambda_{\alpha}\left\langle\chi_{\alpha}\right|$, where $\left|\chi_{\alpha}\right\rangle$ is an $s$-wave state of the pair $\alpha$. Note that each pair potential supports at most a single bound state (i.e., $N_{\alpha_{\mathrm{B}}}=1$ ). We use the identical-particle version of this model to test the arbitrary nature of pseudochannels used in the approximate theory discussed in the previous section. We take $\left\langle\mathbf{p}_{\alpha} \mid \chi_{\alpha}\right\rangle=\left(\beta^{2}+p_{\alpha}^{2}\right)^{-1}$. The parameters of this model are taken from nuclear physics, where it serves as a prototype of $n+d$ scattering [2]. Accordingly, we take the particle masses to be equal to nucleon mass, and set $\hbar=1$ and $m_{\alpha}=1, \alpha=1,2,3$. Taking the unit of length as $\mathrm{fm}$, the resulting units for momentum and energy are $\mathrm{fm}^{-1}$ and $\mathrm{fm}^{-2}$, respectively. We took $\beta=1.444 \mathrm{fm}^{-1}$, and $\lambda$ was chosen to give the bound state energy for the deuteron, $\varepsilon=-0.0537 \mathrm{fm}^{-2}$.

We further restrict our attention to zero total-angularmomentum state, for which the $K$-matrix version of Eq.(17) reads

$\mathcal{K}^{\beta n^{\prime}, \alpha n}\left(q^{\prime}, \bar{q}_{n}\right)=\mathcal{V}^{\beta n^{\prime}, \alpha n}\left(q^{\prime}, \bar{q}_{n}\right) \sum_{\gamma} \sum_{n^{\prime \prime}} \mathrm{P} \int q^{\prime \prime 2} \mathrm{~d} q^{\prime \prime} \mathcal{V}^{\beta n^{\prime}, \gamma n^{\prime \prime}}\left(q^{\prime}, q^{\prime \prime}\right)\left(E-e_{\gamma n^{\prime \prime}}-q^{\prime \prime 2} / 2 \nu_{\gamma}\right)^{-1} \mathcal{K}^{\gamma n^{\prime \prime}, \alpha n}\left(q^{\prime \prime}, \bar{q}_{n}\right)$

where $\mathrm{P}$ indicates principal-value integral, $E=e_{n}+\bar{q}_{n}^{2} / 2 \nu_{\alpha}$, and

$$
\mathcal{V}^{\beta n^{\prime}, \gamma n}\left(q^{\prime}, q\right)=\frac{1}{4 \pi} \int d \hat{q}^{\prime} \int d \hat{q} \mathcal{V}^{\beta n^{\prime}, \gamma n}\left(\mathbf{q}^{\prime}, \mathbf{q}\right)
$$

In $\mathcal{V}^{\beta n^{\prime}, \gamma n}\left(\mathbf{q}^{\prime}, \mathbf{q}\right)$, the momentum arguments $\mathbf{q}^{\prime}$ and $\mathbf{q}$ are the Jacobi momenta for rearrangements $\beta$ and $\gamma$, respectively. The transition matrix elements are obtained from

$$
\mathcal{A}^{\beta n^{\prime}, \alpha n}\left(q^{\prime}, \bar{q}_{n}\right)=\mathcal{K}^{\beta n^{\prime}, \alpha n}\left(q^{\prime}, \bar{q}_{n}\right)-\sum_{\gamma} \sum_{n^{\prime \prime}=1}^{N_{\gamma}^{\text {open }}} \mathcal{K}^{\beta n^{\prime}, \gamma n^{\prime \prime}}\left(q^{\prime}, \bar{q}_{n^{\prime \prime}}\right)\left(i \pi \nu_{\gamma} \bar{q}_{n^{\prime \prime}}\right) \mathcal{A}^{\gamma n^{\prime \prime}, \alpha n}\left(\bar{q}_{n^{\prime \prime}}, \bar{q}_{n}\right)
$$

where $N_{\gamma}^{\text {open }}$ is the number of channels in rearrangement $\gamma$ satisfying $E \geq e_{\gamma n}$.

Assuming that the subspaces $\mathcal{S}_{\alpha}, \alpha=1,2,3$, are constructed to respect the identity of the three particles, Eq. (35) can be block-diagonalized according to the irreducible representations of the permutation group $S_{3}$ [25]. For the present model with all angular momenta restricted to zero, the totally symmetric (S) and mixed-symmetry (E) matrix elements can be defined via

$$
\begin{aligned}
& \mathcal{O}_{\mathrm{S}}^{n^{\prime} n}\left(q^{\prime}, q\right)=\mathcal{O}^{1 n^{\prime}, 1 n}\left(q^{\prime}, q\right)+2 \mathcal{O}^{2 n^{\prime}, 1 n}\left(q^{\prime}, q\right), \\
& \mathcal{O}_{\mathrm{E}}^{n^{\prime} n}\left(q^{\prime}, q\right)=\mathcal{O}^{1 n^{\prime}, 1 n}\left(q^{\prime}, q\right)-\mathcal{O}^{2 n^{\prime}, 1 n}\left(q^{\prime}, q\right)
\end{aligned}
$$

where $\mathcal{O}=\mathcal{A}, \mathcal{K}$, or $\mathcal{V}$, and rearrangement 1 serves as the reference partition. The symmetry-adapted versions of (35) and (37) are

$$
\begin{aligned}
& \mathcal{K}_{\tau}^{n^{\prime} n}\left(q^{\prime}, \bar{q}_{n}\right)=\mathcal{V}_{\tau}^{n^{\prime} n}\left(q^{\prime}, \bar{q}_{n}\right)+\sum_{n^{\prime \prime}} \mathrm{P} \int q^{\prime \prime 2} \mathrm{~d} q^{\prime \prime} \mathcal{V}_{\tau}^{n^{\prime} n^{\prime \prime}}\left(q^{\prime}, q^{\prime \prime}\right)\left(E-e_{1 n^{\prime \prime}}-q^{\prime \prime 2} / 2 \nu_{1}\right)^{-1} \mathcal{K}_{\tau}^{n^{\prime} n}\left(q^{\prime \prime}, \bar{q}_{n}\right) \\
& \mathcal{A}_{\tau}^{n^{\prime} n}\left(q^{\prime}, \bar{q}_{n}\right)=\mathcal{K}_{\tau}^{n^{\prime} n}\left(q^{\prime}, \bar{q}_{n}\right)-\sum_{n^{\prime \prime}=1}^{N_{1}^{\text {open }}} \mathcal{K}_{\tau}^{n^{\prime} n^{\prime \prime}}\left(q^{\prime}, \bar{q}_{n^{\prime \prime}}\right)\left(i \pi \nu_{1} \bar{q}_{n^{\prime \prime}}\right) \mathcal{A}_{\tau}^{n^{\prime \prime} n}\left(\bar{q}_{n^{\prime \prime}}, \bar{q}_{n}\right)
\end{aligned}
$$


where the symmetry index $\tau$ is either $\mathrm{S}$ or $\mathrm{E}$. Of course, this symmetrization procedure is equivalent to first decomposing $\mathcal{S}$ and $\mathcal{S}$ as into irreducible-representation subspaces, and then formulating the approximate theory for each symmetry $\tau$ separately by using the pair of spaces $\left(\mathcal{S}_{\tau}, \mathcal{S}_{\tau}^{\text {as }}\right)$.

Note that, for each irreducible representation, our model has just one open channel in the energy interval $-0.0537<E<0$. In this elastic regime, our normalization convention implies $\mathcal{A}_{\tau}^{11}=-\left(\pi \nu_{1} q_{1}\right)^{-1} \sin \delta_{\tau} e^{i \delta_{\tau}}$. Changing from momentum normalization to energy normalization, we define $\overline{\mathcal{A}}_{\tau}^{n^{\prime} n}=\nu_{1} \sqrt{q_{n^{\prime}} q_{n}} \mathcal{A}_{\tau}^{n^{\prime} n}$. The $S$-matrix elements are then given as $S_{\tau}^{n^{\prime} n}=\delta_{n^{\prime} n}-$ $2 \pi i \overline{\mathcal{A}}_{\tau}^{n^{\prime} n}$.

The coupled set of integral equations (37) are first regularized by a subtraction method [26], and the resulting set of nonsingular equations are solved with quadrature discretization. The results presented in Tables II and III were obtained using 48-64 quadrature points. Sensitivity of the results to further increase in the number of quadrature points is typically less than 0.0005 .

The reference solutions in Tables II and III were obtained by solving the Faddeev equations. The solution technique employed is the Schwinger variational method using piecewise interpolation functions of relative momentum as basis functions. As mentioned in the Introduction, the kernel of the momentum-space Faddeev equations for $E>0$ has logarithmic singularities, which were handled using a subtraction technique [27]. The reference results are stable to better than \pm 0.0005 .

\section{B. Pseudostates}

The primitive two-particle subspaces are spanned by basis functions

$u_{n}(\mathbf{x})=C_{n} e^{-\zeta x} L_{(n-1)}^{(2)}(2 \zeta x) Y_{00}(\hat{\mathbf{x}}), \quad n=1,2, \ldots, N_{\max }$

where $L_{(n)}^{(m)}$ denote an associated Laguerre polynomial, and $C_{n}$ is a normalization constant. Specifying $\zeta$ and $N_{\max }$ determines the primitive two-particle subspace $\tilde{s}_{\alpha}\left(\zeta, N_{\max }\right)$. A set of $N_{\max }-1$ pseudostates are obtained by diagonalizing the two-particle Hamiltonian on $\tilde{s}_{\alpha}\left(\zeta, N_{\max }\right)$. Use of the lowest $N_{\alpha}$ states for each of the three rearrangements then yields a full approximation space $\mathcal{S}$ characterized by the set of parameters $\left\{\zeta_{\alpha}, N_{\alpha, \max }, N_{\alpha}, \alpha=1,2,3\right\}$. For three identical particles, the full approximation space can be denoted as $\mathcal{S}\left(\zeta, N_{\max }, N\right)$.

Using different $\left(\zeta, N_{\max }\right)$ combinations, different sets of pseudostates are generated. The six combinations we used are shown in Table I. To indicate the rather different nature of these six sets, the number of pseudostates with energies less than $0.1,0.5,1.0$, and $2.0 \mathrm{fm}^{-2}$ are also shown in the same table. Uses of different $N_{\max }$ with a given value of $\zeta$ yield quite different spectra of pseudostate energies. With larger $N_{\max }$, more of the pseudostates are crowded at the lower energies. On the other hand, among the three sets with $N_{\max }=30$, the one with $\zeta=0.7$ (set $A$ ) gives the densest pseudostate spectrum at the lower energy range.

\section{Discussion of results}

The calculations were done at four different energies, $E=0.1,0.5,1.0$, and $2.0 \mathrm{fm}^{-2}$, at which the Faddeev calculations give total breakup probabilities of 0.136 , $0.692,0.919$, and 0.835 for the totally-symmetric case, and $0.013,0.191,0.226,0.148$ for the mixed-symmetry case, respectively. Thus, the breakup channel is clearly dominant in the symmetric case and is non-negligible in the mixed-symmetry case.

A pseudostate calculation (for the identical particle case) is characterized by the approximation space $\mathcal{S}_{\tau}\left(\zeta, N_{\max }, N\right)$, where $\tau=\mathrm{E}$, or $\mathrm{S}$, and $N\left(\leq N_{\max }\right)$ is the actual number of pseudostates used. Results for 14 different $\left(\zeta, N_{\max }, N\right)$ combinations are given in Tables II and III. Results are grouped, first, according to $\zeta$. For each value of $\zeta$, the results are further divided into subgroups for different $N_{\max }$. A limited number of calculations using different $N$ for a given $\left(\zeta, N_{\max }\right)$ combination were also performed to probe the convergence behavior as $\mathcal{S}\left(\zeta, N_{\max }, N\right) \rightarrow \mathcal{S}\left(\zeta, N_{\max }, N_{\max }\right)$

Comparing the results on different maximal approximation spaces $\mathcal{S}\left(\zeta, N_{\max }, N_{\max }\right)$, we find that the results are all similar and the agreement with the reference results are quite satisfactory. The amplitudes $\overline{\mathcal{A}}_{\tau}^{11}$ for transitions between true bound states are predicted quantitatively. Thus, by unitarity of the approximate theory, the total breakup probabilities are also predicted quite accurately. It is noteworthy that the significantly different spectral densities of the different pseudostate sets do not seem to have any serious consequence. Apparently, to ob-

TABLE I. Basis parameters $\zeta$ and $N_{\max }$ for the different sets of pseudostates used. Also shown are the number of open pseudochannels at four different values of the total energy $E$.

\begin{tabular}{lcccccc}
\hline \hline & & \multicolumn{5}{c}{ Number of open pseudochannels } \\
Set & $\zeta$ & $N_{\max }$ & $E=0.1$ & $E=0.5$ & $E=1.0$ & $E=2.0$ \\
\hline$A$ & 0.7 & 30 & 7 & 14 & 18 & 21 \\
$B$ & 0.7 & 20 & 5 & 9 & 12 & 14 \\
$C$ & 1.4 & 30 & 3 & 8 & 11 & 14 \\
$D$ & 1.4 & 20 & 2 & 5 & 7 & 7 \\
$E$ & 1.4 & 15 & 1 & 4 & 5 & 11 \\
$F$ & 2.0 & 30 & 2 & 6 & 8 & 9 \\
\hline \hline
\end{tabular}


TABLE II. Results of pseudostate-augmented CRC calculations with different sets of pseudostates for the totally symmetric irreducible representation. Here $\overline{\mathcal{A}}_{\mathrm{S}}$ stands for $\overline{\mathcal{A}}_{\mathrm{S}}^{n^{\prime} n}$ with $n=n^{\prime}=1$, and $N$ is the number of pseudostates used in a given calculation.

\begin{tabular}{|c|c|c|c|c|c|c|c|c|c|}
\hline \multirow[b]{2}{*}{ Set } & \multirow[b]{2}{*}{$N$} & \multicolumn{2}{|c|}{$E=0.1$} & \multicolumn{2}{|c|}{$E=0.5$} & \multicolumn{2}{|c|}{$E=1.0$} & \multicolumn{2}{|c|}{$E=2.0$} \\
\hline & & $\overline{\operatorname{Re} \mathcal{A}_{\mathrm{S}}}$ & $\overline{\operatorname{Im} \mathcal{A}_{\mathrm{S}}}$ & $\overline{\operatorname{Re} \overline{\mathcal{A}}_{\mathrm{S}}}$ & $\overline{\operatorname{Im} \mathcal{A}_{\mathrm{s}}}$ & $\overline{\operatorname{Re} \mathcal{A}_{\mathrm{S}}}$ & $\overline{\operatorname{Im} \overline{\mathcal{A}}_{\mathrm{S}}}$ & $\overline{\operatorname{Re} \overline{\mathcal{A}}_{\mathrm{S}}}$ & $\operatorname{Im} \mathcal{A}_{\mathrm{s}}$ \\
\hline \multirow[t]{3}{*}{$A$} & 15 & 0.0368 & -0.0192 & 0.0774 & -0.1968 & -0.0061 & -0.2359 & -0.1086 & -0.1639 \\
\hline & 20 & 0.0281 & -0.0136 & 0.0820 & -0.1779 & 0.0015 & -0.2059 & -0.0721 & -0.1467 \\
\hline & 30 & 0.0059 & -0.0129 & 0.0882 & -0.1644 & 0.0093 & -0.2041 & -0.0630 & -0.1517 \\
\hline$B$ & 20 & 0.0050 & -0.0099 & 0.0837 & -0.1686 & 0.0110 & -0.2038 & -0.0712 & -0.1483 \\
\hline \multirow[t]{4}{*}{$C$} & 10 & 0.0309 & -0.0157 & 0.0800 & -0.1873 & -0.0060 & -0.2200 & 0.0808 & -0.0985 \\
\hline & 15 & 0.0214 & -0.0160 & 0.0800 & -0.1652 & 0.0036 & -0.2056 & -0.0693 & -0.1473 \\
\hline & 20 & 0.0104 & -0.0121 & 0.0874 & -0.1666 & 0.0083 & -0.2032 & -0.0632 & -0.1499 \\
\hline & 30 & 0.0052 & -0.0115 & 0.0887 & -0.1646 & 0.0123 & -0.2035 & -0.0604 & -0.1504 \\
\hline$D$ & 20 & 0.0058 & -0.0117 & 0.0889 & -0.1639 & 0.0086 & -0.2044 & -0.0641 & -0.1517 \\
\hline$E$ & 15 & 0.0059 & -0.0104 & 0.0874 & -0.1636 & 0.0128 & -0.2043 & -0.0616 & -0.1536 \\
\hline \multirow[t]{4}{*}{$F$} & 10 & -0.0577 & -0.0387 & 0.0834 & -0.1755 & 0.0004 & -0.2038 & -0.0743 & -0.1833 \\
\hline & 15 & 0.0117 & -0.0118 & 0.0867 & -0.1686 & 0.0072 & -0.2020 & -0.0659 & -0.1499 \\
\hline & 20 & 0.0086 & -0.0116 & 0.0871 & -0.1677 & 0.0076 & -0.2015 & -0.0639 & -0.1496 \\
\hline & 30 & 0.0059 & -0.0112 & 0.0878 & -0.1648 & 0.0088 & -0.2020 & -0.0675 & -0.1498 \\
\hline \multicolumn{2}{|c|}{ Faddeev } & 0.0059 & -0.0113 & 0.0880 & -0.1643 & 0.0099 & -0.2033 & -0.0639 & -0.1505 \\
\hline
\end{tabular}

TABLE III. Results of pseudostate-augmented CRC calculations with different sets of pseudostates for the mixed (E) irreducible representation. Here $\overline{\mathcal{A}}_{\mathrm{E}}$ stands for $\overline{\mathcal{A}}_{\mathrm{E}}^{n^{\prime} n}$ with $n=n^{\prime}=1$, and $N$ is the number of pseudostates used in a given calculation.

\begin{tabular}{|c|c|c|c|c|c|c|c|c|c|}
\hline \multirow[b]{2}{*}{ Set } & \multirow[b]{2}{*}{$N$} & \multicolumn{2}{|c|}{$E=0.1$} & \multicolumn{2}{|c|}{$E=0.5$} & \multicolumn{2}{|c|}{$E=1.0$} & \multicolumn{2}{|c|}{$E=2.0$} \\
\hline & & $\operatorname{Re} \overline{\mathcal{A}}_{\mathrm{E}}$ & $\overline{\operatorname{Im} \mathcal{A}_{\mathrm{E}}}$ & $\overline{\operatorname{Re} \overline{\mathcal{A}}_{\mathrm{E}}}$ & $\overline{\operatorname{Im} \mathcal{A}_{\mathrm{E}}}$ & $\overline{\operatorname{Re} \mathcal{A}_{\mathrm{E}}}$ & $\operatorname{Im} \overline{\mathcal{A}}_{\mathrm{E}}$ & $\operatorname{Re} \overline{\mathcal{A}}_{\mathrm{E}}$ & $\operatorname{Im} \overline{\mathcal{A}}_{\mathrm{E}}$ \\
\hline \multirow[t]{3}{*}{$A$} & 15 & -0.0538 & -0.3054 & -0.1423 & -0.1766 & -0.1227 & -0.0884 & -0.0708 & -0.0239 \\
\hline & 20 & -0.0481 & -0.3052 & -0.1423 & -0.1766 & -0.1224 & -0.0915 & -0.0744 & -0.0327 \\
\hline & 30 & -0.0358 & -0.3112 & -0.1422 & -0.1776 & -0.1221 & -0.0917 & -0.0746 & -0.0328 \\
\hline$B$ & 20 & -0.0414 & -0.3101 & -0.1418 & -0.1771 & -0.1198 & -0.0918 & -0.0752 & -0.0320 \\
\hline \multirow[t]{4}{*}{$C$} & 10 & -0.0407 & -0.3121 & -0.1438 & -0.1788 & -0.1231 & -0.0899 & -0.0752 & -0.0294 \\
\hline & 15 & -0.0405 & -0.3122 & -0.1422 & -0.1777 & -0.1227 & -0.0916 & -0.0747 & -0.0327 \\
\hline & 20 & -0.0404 & -0.3122 & -0.1422 & -0.1776 & -0.1227 & -0.0914 & -0.0751 & -0.0326 \\
\hline & 30 & -0.0404 & -0.3123 & -0.1424 & -0.1776 & -0.1226 & -0.0915 & -0.0750 & -0.0326 \\
\hline$D$ & 20 & -0.0413 & -0.3115 & -0.1424 & -0.1780 & -0.1218 & -0.0920 & -0.0747 & -0.0333 \\
\hline$E$ & 15 & -0.0408 & -0.3126 & -0.1425 & -0.1783 & -0.1222 & -0.0918 & -0.0784 & -0.0309 \\
\hline \multirow[t]{4}{*}{$F$} & 10 & -0.0407 & -0.3120 & -0.1421 & -0.1774 & -0.1227 & -0.0910 & -0.0750 & -0.0319 \\
\hline & 15 & -0.0406 & -0.3121 & -0.1425 & -0.1770 & -0.1229 & -0.0908 & -0.0751 & -0.0334 \\
\hline & 20 & -0.0405 & -0.3120 & -0.1420 & -0.1756 & -0.1225 & -0.0911 & -0.0730 & -0.0324 \\
\hline & 30 & -0.0386 & -0.3122 & -0.1422 & -0.1775 & -0.1231 & -0.0916 & -0.0747 & -0.0331 \\
\hline \multicolumn{2}{|c|}{ Faddeev } & -0.0406 & -0.3121 & -0.1420 & -0.1775 & -0.1226 & -0.0915 & -0.0747 & -0.0328 \\
\hline
\end{tabular}


tain the correct rearrangement amplitudes, it is enough to have a few open pseudochannels that provide an outlet for the flux that would have appeared as the breakup flux in an exact treatment. The main role for the rest of the pseudostates is in enlargening the function space and, thus, improving the approximation of the wave function at short distances (i.e., the strong interaction region).

The results also show a (nonmonotonic) convergence as the sequence $\mathcal{S}\left(\zeta, N_{\max }, N\right)$ of approximation spaces approaches $\mathcal{S}\left(\zeta, N_{\max }, N_{\max }\right)$, for fixed $\zeta$ and $N_{\max }$. The convergence rates, however, are different for different combinations of $\zeta$ and $N_{\max }$. Although we have not attempted to find the optimal values for the basis parameters, the limited number of convergence studies conducted for the sets $A, C$ and $F$, indicate that set $A$, which has the most number of open pseudochannels at all four energies, shows the slowest convergence. This implies that it is not just the number of open pseudochannels that matters, but a sufficient number of high-energy pseudostates are also needed.

\section{CONCLUSIONS}

In this paper, techniques adopted from the twoHilbert-space approach have been used to derive the pseudochannel-augmented CRC equations. Usefulness of this approach is especially evident when an approximation space is constructed from asymptotic subspaces that are not mutually orthogonal. Our formulation, of course, subsumes the standard CRC method. Thus, the relationship of the effective transition operators of the CRC method to the post-form transition operators of the multichannel scattering theory has also been established.

The present results further corroborate the earlier findings $[10,19]$ that the breakup can be simulated with pseudoreaction channels. The arbitrariness involved in the choice of the pseudochannels does not represent a problem, although it can be of practical concern as far as convergence rates are concerned. Provided a sufficient number of pseudostates are employed, the reaction amplitudes (hence, the total breakup probabilities) are predicted quantitatively. In this connection, our results show that to obtain optimal results, the psedostate energy spectrum should be balanced in its low- and highenergy members. A large number of open pseudochannels does not necessarily give the best results, unless a certain number of closed high-energy states are also included.

Our results, however, are presently limited to the rearrangement amplitudes, and, by unitarity, to total breakup probability. How the total breakup probability is divided between the two degrees of freedom ( e.g., $\mathbf{q}_{\alpha}$ and $\mathbf{p}_{\alpha}$ ) involved in breakup is a more difficult question. For a collison starting in a two-fragment channel $\alpha$, the breakup transition operator is $U_{0 \alpha}^{(+)}(E)=$ $V G(E) G_{\alpha}(E)^{-1}=V+V G(E) V^{\alpha}$. Breakup amplitude $\left\langle\mathbf{p}_{\beta} \mathbf{q}_{\beta}\left|U_{0 \alpha}^{(+)}(E)\right| \varphi_{\alpha n} \overline{\mathbf{q}}_{\alpha n}\right\rangle$ can also be expressed in terms of the rearrangement operators [2]:

$$
\left\langle\mathbf{p}_{\beta} \mathbf{q}_{\beta}\left|U_{0 \alpha}^{(+)}(E)\right| \varphi_{\alpha n} \overline{\mathbf{q}}_{\alpha n}\right\rangle=\left\langle\varphi_{\beta \mathbf{p}}^{(-)} \mathbf{q}_{\beta}\left|U_{\beta \alpha}^{(+)}(E)\right| \varphi_{\alpha n} \overline{\mathbf{q}}\right\rangle,
$$

where $E=e_{\alpha n}+\bar{q}_{\alpha n}^{2} / 2 \nu_{\alpha}=p_{\beta}^{2} / 2 \mu_{\beta}+q_{\beta}^{2} / 2 \nu_{\beta}$, and the partition index $\beta$ in these expressions can be taken as 1,2 , or 3 . Given that a pseudostate $\left|\phi_{\alpha n}\right\rangle$ is an approximation to $\varphi_{\alpha p_{n}}^{(-)}$, with $p_{n}=\sqrt{2 \mu_{\alpha} e_{\alpha n}}$, it would be tempting to consider $w_{\beta n^{\prime}} \mathcal{A}^{\beta n^{\prime}, 11}\left(\overline{\mathbf{q}}_{n^{\prime}}, \overline{\mathbf{q}}_{11}\right)$ for $n^{\prime} \neq 1$ as an approximation to the breakup amplitude $\left\langle\varphi_{\beta p_{n^{\prime}}}^{(-)} \overline{\mathbf{q}}_{\beta n^{\prime}}\left|U_{\beta 1}^{(+)}(E)\right| \varphi_{11} \overline{\mathbf{q}}_{11}\right\rangle$. This view might be tenable in a single-rearrangement system [8-10], for breakup then can uniquely be associated with the continuum of just one pair. However, when there are three rearrangements, and pseudostates for all rearrangements are used ( as we do), the breakup is represented by three different pieces of the total wave function, and the idea of the quadrature discretization of the breakup continuum is no longer tenable.

As an alternative, one could consider approximating $U_{\beta \alpha}^{(+)}$in Eq. (43) with $U_{\beta, \alpha}^{(+) \pi}$. The required matrix element can then be calculated from the pseudochannel amplitudes using Eq. (22). Whether this scheme will provide a means to extract reliable breakup amplitudes is an open question. Since the approximate theory violates the exact breakup boundary conditions, it is plausible that it will never yield accurate state-to-state breakup amplitudes . Presumably, the success of the method in predicting the rearrangement amplitudes is due to working with a large enough approximation space capable of describing the total wave function in the interior, and having enough open pseudochannels to divert the breakup flux from going into reaction channels, as, e.g, would occur with the usual CRC expansion above the breakup.

Finally, we note that the equations derived in Sec. III C have the same structure as the equations in the approximation theory of Chandler and Gibson [20,21]. Of course, the treatment of the breakup channel is different. As a result, their convergence proof does not apply to the present case, since projectors of the pseudochannels do not commute with the asymptotic Hamiltonians, implying that the exact breakup boundary conditions are violated. It is not clear in what sense an approximate scattering theory involving approximate boundary conditions converges toward the exact theory. Clearly, despite empirical successes, to fully exploit the pseudochannel idea, an answer to this question is needed.

\section{ACKNOWLEDGMENT}

This work has been supported by the Turkish National Research Council (TUBITAK) through Grant No. TBAG-1088. 
[1] L.D. Faddeev, Zh. Eksp. Teor. Fiz. 39, 1459 (1960) [Sov. Phys.-JETP 12, 1014 (1961)]; E.O.Alt, W. Grassberger, and W. Sandhas, Nucl. Phys. B2, 167 (1967).

[2] See, e.g., W.Glockle, The Quantum Mechanical Few Body Problem (Springer, Berlin, 1983).

[3] N.M. Larson and J.H. Hetherington, Phys. Rev. C 9, 699 (1974); T.E. Mdlalose, H. Fiedeldey, and W. Sandhas, Nucl. Phys. A457, 273 (1986).

[4] For a discussion of breakup boundary conditions, see, e.g., S.P. Merkuriev, C. Gignoux, and A. Laverne, Ann. Phys. (NY) 99, 30 (1976); W. Glockle, Z. Phys. 271, 31 (1974).

[5] Z.C. Kuruoglu and F.S. Levin, Phys.Rev. C 36, 49 (1987); W. Glockle, ibid. 37, 6 (1988).

[6] J.L. Friar, B. F. Gibson, G. Berthold, W. Glockle, Th. Cornelius, H. Witala, J. Haidenbauer, Y. Koike, G.L. Payne, L.A. Tjon, and W.M. Kloet, Phys. Rev. C 42, 1838 (1990).

[7] J. A. Kaye and A. Kuppermann, Chem. Phys. Lett. 78, 546 (1981).

[8] See,e.g., W. P. Reinhardt, Comput. Phys. Commun. 17, $1(1979)$, and references therein.

[9] E.W. Knapp and D.J. Diestler, J. Chem. Phys. 67, 4969 (1977);

[10] G. Rawitscher and R.Y. Rasoanaivo, Phys. Rev. C 27, 1078 (1983); 39, 1709 (1989).

[11] For an explicit quadrature discretization of breakup in single-rearrangement models, see Y. Sakuragi, M. Yahiro, and M. Kamimura, Prog. Theor. Phys. 65, 2051 (1981); M. Yahiro, Y. Iseri, M. Kamimura, and M. Nakona, Phys. Lett. 141B, 19 (1984).

[12] P.N. Shen, Y.C. Tang, Y. Fujiwara, and H. Kanada, Phys. Rev. C 31, 2001 (1985).

[13] For a review of the CRC method in the context of nuclear reactions, see Y.C. Tang, M. LeMere, and D.R. Thomp- son, Phys. Rep. 47, 167 (1978). Similar approaches for chemical reactions are described in W.H. Miller, J. Chem. Phys 50, 407 (1969); D.W. Schwenke, D.G. Truhlar, and D.J. Kouri, ibid. 86, 27 (1987).

[14] See, e.g., J. Z. H. Zhang and W.H. Miller, J. Chem. Phys. 88, 4454 (1988); J.Z.H. Zhang, D.J. Kouri, K. Haug, D.W. Schwenke, Y. Shima, and D.G. Truhlar, ibid. 88, 2492 (1988).

[15] Z.C. Kuruoglu, Phys. Rev A 42, 6314 (1990).

[16] C. Chandler, and A. Gibson, J.Math.Phys. 14, 1328 (1973); 18, 2336 (1977).

[17] Gy. Bencze, C. Chandler, and A.G. Gibson, Nucl. Phys. A390, 461 (1982).

[18] M.C. Birse and E.F. Redish, Nucl.Phys. A406, 149 (1983).

[19] Z.C. Kuruoglu and F.S. Levin, Phys.Rev.Lett. 48, 899 (1982); Ann. Phys. (NY) 163, 120 (1985); Z.C. Kuruoglu, Phys. Rev. C 43, 1061 (1991).

[20] A.G. Gibson and C. Chandler, J. Math. Phys. 25, 1841 (1984); J. Funct. Anal. 52, 80 (1983).

[21] A. G. Gibson and C. Chandler, J. Math. Phys. 30, 1533 (1989).

[22] See, e.g., J.T. Broad, Phys. Rev. A 26, 3078 (1982); 18, 1012 (1978), and references therein.

[23] J.R.Taylor, Scattering Theory (Wiley, New York, 1972).

[24] D. J. Kouri and D. K. Hoffmann (private communication).

[25] See, e.g., J.Z.H. Zhang and W.H. Miller, J. Chem. Phys. 91, 1528 (1989).

[26] The subtraction method used is described in the appendix of Z.C. Kuruoglu and D.A. Micha, J.Chem.Phys. 80, 4262 (1984).

[27] The subtraction method used for the logarithmic singularities is similar to the one given by W. M. Kloet and J. A. Tjon, Ann. Phys. (NY) 79, 407 (1973). 